\title{
Effect of Depression on Function of Natural Killer Cell in Patients with Leukemia
}

\author{
Y. NIE ${ }^{1}$, B. JU' 1 Y. MIAO ${ }^{2}$, H. ZHANG ${ }^{1}$ AND J. SU ${ }^{3^{* *}}$ \\ ${ }^{1}$ Department of Immune, Basic Medical College of Mu Dan Jiang Medical University, Mudanjiang City 157011, ${ }^{2}$ Department \\ of Psychopsychology, Hongqi Hospital of Mudanjiang Medical University, Mudanjiang City 157011, ${ }^{3}$ Department of Medical \\ Psychology, School of Public Health of Mudanjiang Medical University, Mudanjiang City 157011, China
}

Nie et al.: Effect of Depression on Function of Natural Killer Cell in Patients with Leukemia

\begin{abstract}
The purpose of this study is to enhance the killing sensitivity of natural killer cells and improve the efficacy of leukemia treatment. In this study, 134 patients with malignant hematological diseases are screened with the Hamilton depression scale (24 items), and 49 patients with leukemia with depression are randomly divided into the intervention group and the control group. In addition to routine medical treatment, the intervention group is treated with antidepressant medications. It was found that there is a correlation between depression and function of natural killer cell. According to this study, the depressed psychology of leukemia patients would reduce the appetite of the patient and lead to the low nutrition of the body, which also affects the quality of sleep. And this kind of influence would aggravate the depression degree of cancer patients, and this kind of negative psychological state would also affect the patients' compliance in the treatment process, so as to affect the final treatment effect of patients. Psychosocial factors are closely related to their own psychological factors, social role environment, nervous system - endocrine system, immune system of the body, and the occurrence and development of tumor itself, as well as their interaction and mutual promotion. Therefore, depression can lower the immunity of leukemia patients, and antidepressant drugs can significantly reduce the level of anxiety and depression in leukemia patients and may improve the immunity of patients to a certain extentn.
\end{abstract}

Key words: leukemia, NK cell, immunity, depression

With the advancement of society, people's living standards are constantly improving, and human health has become a focus of attention. Depressive disorder is one of the most common psychological diseases ${ }^{[1-3]}$. According to foreign statistics, the prevalence of depression in the general population is about $6 \%$, anxiety state accounts for $2 \%-5 \%$, and the prevalence of depression in hospitalized patients or cancer patients is at least 3-4 times higher than that of the general population, reaching $22-24 \%{ }^{[4]}$. Current studies of

*Address for correspondence 
cancer patients, including leukemia, have found that about one in five develop chronic depression. Depression and anxiety add unnecessary pain to the sufferer, and many studies have shown that depressed mood can negatively affect the outcome of the disease. From a psychological point of view, the psychological response of cancer patients is actually caused by strong stress ${ }^{[5]}$. A study of mental disorders in cancer patients found that 13 percent of those with malignant tumors had major depression, and 4 percent had anxiety disorders or even suicidal behavior ${ }^{[6]}$. Therefore, psychological intervention for cancer patients plays an increasingly important role in the clinical treatment of cancer. In recent years, many researchers have studied the effect of depression on the function of NK cells $s^{[7]}$. There is a close and complex relationship between depression and the immune system, which is reflected in the following aspects: firstly, most patients with depression have immune activation phenomenon, which can be improved after long-term treatment with antidepressants $^{[8]}$. Secondly, some cytokines can activate the HPA axis, and patients treated with cytokines can show depressive behavior ${ }^{[9]}$. Thirdly, some patients with immune disorders are often accompanied by depressive symptoms. These are the mechanisms that can be observed in patients with depression and are well recognized. In the aspect of cellular immunity, the study on the relationship between the activity of $\mathrm{T}$ cells and NK cells in cancer patients with depression found that there was a significant negative correlation between the severity index of depression and T4 / T8, and there was also a certain negative correlation between them and NK cells ${ }^{[10]}$. In terms of humoral immunity, depression may cause changes in the content and activity of various cytokines. In this study, for patients with malignant hematological diseases with strong homogeneity of leukemia, the incidence of depressive disorder and the effect of antidepressant treatment on the function of NK cells and immune function in patients with malignant hematological diseases and depressive disorders are investigated. NK cells in the blood of leukemia patients and other important immune cells. NK cells are described as "large granule lymphocytes", which contain particles of perforin and granzyme that are important weapons against target cells. When NK cells come into contact with target cells, they are activated and release perforin and granzyme through degranulation effect. Among them, perforin polymerizes on the surface of the target cell to form pores, allowing $\mathrm{Na}^{+}$ions and water to enter the target cell, while $\mathrm{K}$ ions and macromolecular substances including proteins flow out of the cell, changing the osmotic pressure of the cell and eventually leading to lysis. Granulozyme can enter target cells and induce apoptosis. In addition, NK cells also express Fas ligand (FasL) and TNF-related apoptosis-inducing ligand (TRAIL) in TNF family members. It can induce apoptosis of target cells after binding to corresponding receptors on the surface of target cells. In addition, membrane TNF-a on the surface of NK cells induces apoptosis of target cells by binding to its ligand ${ }^{[11]}$. The killing function of NK cells against tumors is not only regulated by IL-2 and other cytokines, but also regulated by the expression of multiple activated and inhibitory receptors on NK cells. When activating or inhibiting receptors on the surface of NK cells are cross-linked with the corresponding ligands, it can deliver activating or inhibitory signals to NK cells though a series of signaling molecules, then regulate expression of related genes through various transcription factors, so as to regulate the cytotoxic activity of NK cells and the secretion of cytokines. A total of 134 patients, including 58 females and 76 males aged between 18 and 79 (49.99 \pm 16.34$)$, are enrolled in the hospital under natural conditions. Clinical diagnosis shows that there are 73 cases of acute leukemia, 18 cases of chronic leukemia, 15 cases of myelodysplastic syndrome, 3 cases of multiple myeloma, and 25 cases of lymphoma. The Hamilton depression scale (24 items) is used for psychological evaluation on the day of admission, and patients with scale of 20 or more are randomly divided into the intervention group and the control group. Except for patients who meet one of the following conditions: patients with a history of mental illness, alcoholism, and drug dependence; patients with a history of brain organic diseases or endocrine diseases; patients with severe cognitive impairment who can't communicate effectively; and patients with severe liver and kidney dysfunction. Grouping results: there are 25 patients in the intervention group and 24 patients in the control group. Among them, 2 people in the intervention group and 1 people in the control group are exfoliated, and the causes of exfoliation are all deaths. The effective cases for completing the experiment are 23 people in each group. In the intervention group, there are 13 males and 10 females aged 27-75 y (49.92 \pm 14.86$)$. In the control group, there are 14 males and 9 females aged between 26 and 66 (46.77 \pm 12.15$)$. And there are no significant differences in the conditions between the two groups. 134 patients are admitted to the hospital in the natural state. After admission, Hamilton depression 
scale (HAMD) is used to evaluate the depression degree of patients with malignant tumors, and blood routine, blood biochemistry and lymphocyte subsets of the patients are measured. T cell subsets are expressed by CD3, CD4, CD8, CD4 / CD8, and NK cells are identified by $\mathrm{CD} 16$ and $\mathrm{CD} 56$ double markers. The assay is performed with the Epics-X L flow cytometer manufactured by Coulter, USA. On the first day after admission, two senior psychiatrists complete the HAMD assessment for qualified patients. Patients with scores greater than or equal to 21 are randomly assigned to the intervention and control groups, and the characteristics and common side effects of drug therapy are introduced to them. In the natural state, 33 patients with lung cancer in the department of oncology in the hospital are treated with antidepressant therapy during hospitalization in the treatment group. The remaining treatments are orally administered with olanzapine $(10 \mathrm{mg} \times 1$ Tablet of zyprexa) at $10 \mathrm{mg} / \mathrm{d}$ for 4 weeks. HAMD is used to evaluate the depression degree of patients with malignant tumors 4 weeks later. Blood routine, blood biochemistry, and lymphocyte subsets are measured in the intervention group. And the results of blood routine, blood biochemistry, and lymphocyte subsets are all based on clinically uniform standards. HAMD of 24 items is selected. Among them, total score: an indicator reflecting the severity of the condition, that is, the lighter the condition, the lower the total score; the worse the illness, the higher the score. And it is a very important piece of information. Factor score: HAMD can be classified into 7 types of factor structure. Anxiety / somatization: it consists of five items, including mental anxiety, physical anxiety, gastrointestinal symptoms, suspected disease, and selfconsciousness. Weight: weight loss. Cognitive obstacles: it consists of six factors, that is, self-guilt, suicide, agitation, disintegration of personality and disintegration of reality, paranoid symptoms, and obsessive-compulsive symptoms. Day and night changes: the change at day and night. Block: it consists of four items, that is, depression, work and interest, block, and sexual symptoms. Sleep disorders: including difficulty falling asleep, insomnia-middle, and early awakening. Feeling of despair: including ability drop feeling, feeling of despair, inferiority, etc. This makes the patient's actual characteristics simpler and clearer. Factor analysis can not only reflect the patient's psychopathological characteristics, but also reflect the clinical outcome of the target symptom group. According to the demarcation score, the total score of more than 35 points may be serious depression; the total score of more than 20 points may be mild or moderate depression; and the score of less than 8 points indicates no depressive symptoms. The evaluation of the scale is as follows. First, application reliability: the raters can achieve a high degree of consistency after strict training. For the evaluation of 70 patients with depression, the reliability between the assessors is 0.90 . Among the 14 cooperative units in China, the consistency between the two assessors is quite good. The reliability coefficient $r$ of the total score is $0.88 \sim$ 0.99 , and the $\mathrm{P}$ values are all less than 0.01 . Second, validity: the total score of HAMD can better reflect the severity of the disease. Domestic data report that the empirical truth coefficient of depression in reflecting the severity of clinical symptoms is 0.92. Third, practicability: the HAMD assessment method is simple and easy to master, which can be used for the assessment of depressive symptoms, bipolar disorder, neurosis and other diseases. SPSS 13.0 statistical software package is used. Paired $t$ test is used for the comparison of normality data in each group, and Wilcoxon's sign rank

TABLE 1: SCORE CHANGES OF INTERVENTION GROUP AND CONTROL GROUP BEFORE AND AFTER TREATMENT $(\bar{X} \pm S)$

\begin{tabular}{lcccc}
\hline \multicolumn{3}{c}{ Number of cases } & HAMDscore \\
\hline & & Before treatment & Second weekend & Fourth weekend \\
Intervention group & 23 & $24.33 \pm 9.23$ & $19.59 \pm 8.77$ & $12.62 \pm 7.75$ \\
control group & 23 & $25.46 \pm 4.76$ & $26.13 \pm 6.83$ & $26.29 \pm 6.36$ \\
\hline
\end{tabular}

TABLE 2: CHANGES OF PLASMA T CELL SUBSETS IN INTERVENTION GROUP AND CONTROL GROUP $(\overline{\mathrm{X}} \pm \mathrm{S})$

\begin{tabular}{lccccc}
\hline & & \multicolumn{2}{c}{ Intervention group } & \multicolumn{2}{c}{ control group } \\
\hline & $\mathrm{n}$ & Before treatment & Fourth weekend & Before treatment & Fourth weekend \\
CD3 & 23 & $57.75 \pm 24.87$ & $68.26 \pm 19.56$ & $55.28 \pm 23.87$ & $54.78 \pm 26.45$ \\
CD4 & 23 & $24.78 \pm 12.34$ & $32.46 \pm 12.72$ & $40.23 \pm 17.25$ & $33.98 \pm 14.78$ \\
CD8 & 23 & $34.59 \pm 10.97$ & $32.21 \pm 10.07$ & $19.67 \pm 13.56$ & $26.39 \pm 12.22$ \\
CD4 / CD8 & 23 & $0.89 \pm 0.26$ & $1.03 \pm 0.14$ & $1.35 \pm 0.22$ & $0.84 \pm 0.32$ \\
\hline
\end{tabular}


test is used for non-normality data. X test is used for counting comparison between the two groups, and Pearson correlation analysis is used for correlation between the two indexes. With the progress of treatment, the HAMD score of the intervention group decrease gradually at the second and fourth weekends, and HAMD scores before treatment are significantly different from those at the second and fourth weekends ( $t$ values are 3.402, 9.722, respectively, $\mathrm{P}<0.01$ ). In the control group, there is no significant difference between the pre-treatment and the second week and the fourth weekend ( $\mathrm{t}$ values are 1.195, 0.960, respectively, $\mathrm{P}>0.05$ ), as shown in Table 1 .

Table 2 shows that CD3, CD4 and CD4 / CD8 in the intervention group are significantly increased at the fourth weekend of antidepressant treatment compared with that before treatment ( $\mathrm{t}$ values are 2.740, 2.811, and 3.019, respectively, and $\mathrm{P}<0.05$ ), and there is no significant change in CD8 ( $\mathrm{t}$ is $0.973, \mathrm{P}>0.05$ ). And there is no significant difference of each item in the control group ( $\mathrm{t}$ values are $0.832,0.901,1.119$ and 0.758 , respectively, $\mathrm{P}>0.05)$. NLR: the difference between the intervention group and the control group

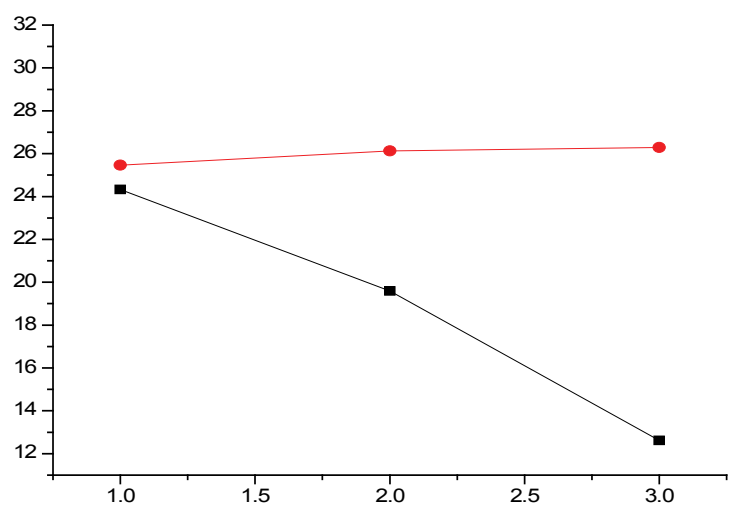

Fig.1: Score changes of intervention group and control group before and after treatment



Fig. 2: Contrast chart of $t$ cell subsets before and after treatment in intervention group

Special Issue 6, 2020 is statistically significant $(\mathrm{Z}=-3.495$ and $\mathrm{P}=0.00)$. CD3: the difference between the intervention group and the control group is statistically significant $(\mathrm{t}=3.567$ and $\mathrm{P}=0.001)$. CD4: the difference between the intervention group and the control group is statistically significant $(\mathrm{t}=-3.237$ and $\mathrm{P}=0.001)$. NK: the difference between the intervention group and the control group is statistically significant $(\mathrm{t}=3.421$ and $\mathrm{P}=0.001)$. B lymphocytes: the difference between the intervention group and the control group is statistically significant $(\mathrm{t}=-1.986$ and $\mathrm{P}=0.056$ ). High-density lipoprotein: the difference between the intervention group and the control group is statistically significant $(\mathrm{t}=2.478$ and $\mathrm{P}=0.021)$. The intervention group have higher CD3, CD4 and NK cells than the control group, which is statistically significant $(\mathrm{P}=0.001, \mathrm{P}=0.001, \mathrm{P}=0.001)$. The control group have higher NLR and $\mathrm{B}$ lymphocytes than the intervention group, which is statistically significant $(\mathrm{P}=0.001$ and $\mathrm{P}=0.056$ ). And the intervention group have better HDL (high density lipoprotein) levels than the control group, which is statistically significant $(\mathrm{t}=2.478$ and $\mathrm{P}=0.021)$. Depression and anxiety can affect the immune function of patients with anxiety and depression by secreting hormones through the hypothalamus and endocrine organs controlled by it. These hormones secreted by the hypothalamus, through the operation of body fluids, can inhibit the body's ability to recognize and kill tumor cells, including NK cells. As an important effector cell against tumor immunity, NK cells can control the growth and spread of various tumors. The function of the immune system is to recognize and defend against foreign proteins, whether they come from pathogens or from the transformation of its own normal cells fig. $1 \& 2$. Therefore, the growth and spread of tumor cells are not only due to tumor-like malignant transformation, but also may be caused by the immune response against foreign proteins. Malignant tumor cells can evade the body's defense through a variety of mechanisms, including transforming the body's t-cell receptor / CD3 complex, or reducing the expression of major histocompatibility antigens on the surface of tumor cells, or secreting inhibitory cytokines. Depression can lead to low proliferation response of lymphocytes during mitosis, decreased activity of NK killer cells, and the change in the number of various white blood cells, thus resulting in low immunity. To sum up, the mechanism of decreased NK cell activity in leukemia patients is explored through the effect of depression on the human immune system. It can be concluded that the application of antidepressants can actually improve the life quality of patients with malignant hematological 
diseases and depression, improve their compliance with the treatment of primary diseases, so as to improve the treatment effect of primary diseases. Since current studies on the biological mechanism of depression and other substances have been found to be associated with depressive mood, it requires further efforts to find laboratory indicators that are directly related to depression degree.

\section{Acknowledgment}

This work was supported by Scientific Research Projects of Basic Scientific Research Business Expenses of Higher Education Institutions in Heilongjiang Province (No. 2018-KYWFMY-0027).

\section{REFERENCES}

1. Sørskaar D, Førre O, Albrechtsen D. Decreased natural killer cell activity versus normal natural killer cell markers in mononuclear cells from patients with smouldering leukemia. Scandinavian J Haematol 2010;37(2): 154-61.

2. Su JP, Liu HF, Zhang HL, He YJ, Nie Y. Effects of different degrees of depression on inflammatory response and immune function in patients with ovarian cancer. J Biol Regul Homeost Agents 2018;32(5): 1225-30.

3. Di Stasio D, Candotto V, Serpico R. Depression and distress in burning mouth syndrome: A case control study. J Biol Regul Homeost Agents, 2018; 32(2 Suppl. 1): 91-95.

4. Currier MB, Nemeroff CB. Depression as a risk factor for cancer: from pathophysiological advances to treatment implications. Annual Rev Med 2014;65(65): 203.

5. Kakoo-Brioso E, Costa L, Ouakinin S. Association of anger expression-out with nk cell counts in colorectal cancer patients. Acta Medica Portuguesa 2018;31(3): 152.

6. Lukomska B, Olszewski WL, Engeset A. The effect of surgery and chemotherapy on blood NK cell activity in patients with ovarian cancer. Cancer 2015;51(3): 465-9.

7. Richter J, Kral V, Pohorska J. Effects of B-Glucan on Natural Killer Cells in Patients Recovering from Cancer Treatment: Clinical Trial. In J Electrochem Sci 2016;2(22): 26-30.

8. Saussez S, Laumbacher B, Chantrain G. Towards Neuroimmunotherapy for Cancer: the Neurotransmitters Glutamate, Dopamine and GnRH-II aug ment substantially the ability of $T$ cells of few Head and Neck cancer patients to perform spontaneous migration, chemotactic migration and migration towards. J Neural Trans 2014;121(8): 1007-27.

9. Farnam A, Majidi J, Nourazar S G. Effect of Anger Patterns and Depression on Serum IgA and NK Cell Frequency. Iranian J Immunol 2016;13(1): 37.

10. Parry HM, Stevens T, Oldreive C. NK cell function is markedly impaired in patients with chronic lymphocytic leukaemia but is preserved in patients with small lymphocytic lymphoma. Oncotarget 2016;7(42): 68513-26.

11. Hsieh CC, Hsiao FH. The effects of supportive care interventions on depressive symptoms among patients with lung cancer: A metaanalysis of randomized controlled studies. Palliat Supp Care 2017;15(6): 1.

This is an open access article distributed under the terms of the Creative Commons Attribution-NonCommercial-ShareAlike 3.0 License, which allows others to remix, tweak, and build upon the work non-commercially, as long as the author is credited and the new creations are licensed under the identical terms

This article was originally published in Special issue on "Trends in therapeutic Management of Various Conditions" Indian J Pharm Sci 2020:82(3) spl issue 6;111-115 